\title{
Un programa de actividades de juego para promover la satisfacción escolar
}

\author{
A game activities program to promote school satisfaction
}

\author{
Pablo Marcos Cancelo \\ Universidade da Coruña
}

\begin{abstract}
Resumen
La educación es un factor fundamental en el desarrollo de todo ser humano. Sin embargo, no es infrecuente que en la infancia y adolescencia se pierda el interés por participar en la escuela. En este contexto, se ha demostrado que el juego es un elemento esencial para el desarrollo significativo y para promover la motivación, convivencia y satisfacción. Por ello, este proyecto tiene por objetivo diseñar un programa de juego para promover una relación positiva alumno-escuela mediante un diseño de medidas repetidas donde se valorarán las diferencias entre un grupo experimental y uno control en las variables estudiadas.

Palabras clave: juegos, convivencia escolar, motivación, satisfacción
\end{abstract}

\section{Introducción}

La educación, es un fenómeno que actúa tanto como agente de socialización, como base de conocimiento y desarrollo de todo ser humano. No obstante, pese a su importancia en el crecimiento de toda persona, no es infrecuente que se produzca un progresivo descenso del interés y motivación de los alumnos a lo largo de la enseñanza primaria y secundaria (Gillet, Vallerand y Lafrenière, 2012).

Como respuesta, diversas investigaciones han tenido lugar para producir un cambio en esta dinámica. En este contexto, se han estudiado los beneficios potenciales de las actividades u ocupaciones lúdicas (el juego) para un desarrollo más enriquecedor y significativo en la infancia y adolescencia (Bundy et al, 2008).

Por ello, el presente estudio tiene por objetivo desarrollar un programa para investigar el potencial del juego para mejorar la convivencia en la escuela, la motivación por asistir y participar en ésta y, en última instancia, la satisfacción escolar.

\section{Fundamentación teórica}

Son muchos los estudios destinados a profundizar en las necesidades que presentan los actuales sistemas de enseñanza y las diferentes formas en las que se puede promover una enseñanza más significativa y ajustada a la época y las premisas sociales.

En este contexto, para conocer cómo es la relación entre un alumno y la escuela resulta preciso estudiar aquellos factores más relevantes en dicha interacción. A saber: la motivación del alumnado, el clima o convivencía escolar, y la satisfacción final hacia la escuela y la enseñanza.

Como expone Tardif (1992) la motivación, entendida comúnmente como el querer hacerlo, puede concebirse como "el compromiso, la participación y la persistencia del alumno en las actividades de clase” (citado en Valenzuela, 2007).

Ésta juega un papel fundamental en la aproximación y persistencia de un individuo hacia una actividad $\mathrm{u}$ ocupación. Además, el tipo de motivación, ya sea intrínseca (interés hacia una actividad por placer) o extrínseca (juicios positivos, aprobación de terceros, etc.), determina la relación futura entre una persona y el centro educativo o el aprendizaje (González, Valle, Nuñez y González-Pienda, 1996). Por consiguiente, cabe señalar que la motivación que se desarrolle durante los primeros años dentro de los sistemas de enseñanza será determinante a lo largo de todo el proceso de aprendizaje académico.

Por su parte, también resulta preciso tomar en consideración factores de carácter contextual a la hora de valorar la relación alumno-escuela. La convivencia o clima escolar, aquellas relaciones interpersonales que se establecen dentro del ámbito escolar y los factores que las producen (Cornejo y Redondo, 2001) son, junto con la motivación, dos elementos esenciales para entender la vinculación de la persona a las ocupaciones académicas, así como su progresivo descenso a lo largo de la enseñanza obligatoria.

Este clima escolar o clima de aula no sólo hace referencia a la interacción entre los iguales (alumnos/as), sino que el profesorado u otros miembros de los centros también forman parte del contexto de participación social que influye y compromete (positiva o negativamente) la calidad de la estancia en el aula. Diversas investigaciones señalan la importancia de las atribuciones causales del propio profesorado sobre los alumnos como un factor más a la hora de valorar su capacidad adaptativa, su rendimiento académico $\mathrm{y}$, en última instancia, su satisfacción escolar (De la Torre y Godoy, 2002).

Finalmente, el factor que determina la vinculación y la significancia final percibida por el alumnado respecto a las actividades u ocupaciones académicas, es la satisfacción escolar. Definida por Cabrera y Galán (2002) como "la coincidencia entre la percepción que el alumnado tiene del contexto educativo y la importancia que éste le da a cada aspecto", la satisfacción escolar puede entenderse como el resultado final de todos aquellos factores que inciden en la relación entre el alumno y la escuela o la enseñanza.

Esta variable adquiere una gran relevancia al encontrarse, a su vez, relacionada con el bienestar subjetivo del individuo, siendo tanto la satisfacción por el ocio como por el trabajo (educación) componentes de dicho bienestar (García, 2002). Además, diversos estudios mantienen que dicha satisfacción se presenta 
como un predictor de una fructífera relación entre el alumno y los procesos educativos (Luna, 2012).

Por su parte, pese a que dichas variables han sido presentadas de forma individual, diversos estudios han corroborado la interrelación e influencia que existe entre éstas. Trabajos como los realizados por Ryan y Deci (2000) analizaron el papel de la participación en la escuela (las condiciones socio-contextuales) en la motivación de los alumnos/as hacia las actividades educativas. Entre sus conclusiones señalaron que las relaciones interpersonales o el apoyo percibido dentro de los centros educativos son factores que favorecen el mantenimiento de una motivación intrínseca y una mayor autodeterminación ante la motivación extrínseca.

A su vez, otras investigaciones también indican la importancia de la relación profesor-alumno (y no sólo entre iguales) para incentivar la motivación de los jóvenes por sus ocupaciones académicas. Dicho estudio recalcó la importancia de realizar reuniones individuales entre docentes y estudiantes para mejorar los vínculos y la calidad de la participación social como medio para una mayor motivación intrínseca (Peetsma y Van der Veen, 2013).

No obstante, no sólo la motivación y el clima de aula están relacionados, estas variables interactúan también con la satisfacción escolar final del alumno. Ante actividades concretas, como son los deberes, se percibe un descenso progresivo en los índices de motivación a lo largo de la enseñanza. Sin embargo, estudios revelan que los alumnos perciben una mayor motivación ante estas tareas cuando su desempeño se realiza de forma conjunta (en un contexto social), y ello repercute en una relación más satisfactoria entre éstos y las actividades educativas (Regueiro, Suárez, Valle, Nuñez y Rosário, 2015).

A mayor escala, en un estudio sobre las variables que influyen o determinan el bienestar de los jóvenes (de entre 11 y 18 años), se señaló a los factores socio-educativos como un elemento relevante a la hora de promover un alto grado de satisfacción infantil y adolescente (Singh y Junnarkar, 2014).

En última instancia, motivación y convivencia son dos variables fundamentales a la hora de estudiar la relación entre el individuo y las ocupaciones educativas, y su satisfacción escolar final. Éstas se influyen mutuamente y ambas condicionan dicha satisfacción. Por consiguiente, resulta preciso tomarlas en consideración si se desea estudiar cómo promover unos procesos de enseñanza y desarrollo funcionales a la par que significativos.

En este contexto, diversas investigaciones han comenzado a estudiar los potenciales beneficios que actividades u ocupaciones significativas en la infancia y adolescencia (como es el juego) pueden tener en aquellas variables de impacto en el ámbito académico.

Concretamente dentro de la escuela o el instituto, se han probado dichos efectos en el aprendizaje de algunas de las materias curriculares. Ejemplo de ello son los estudios de Huang, Huang y Wu (2014) sobre el uso de juegos digitales o videojuegos en clases de matemáticas durante la Enseñanza Primaria. Sus resultados concluyen que tanto los niveles de motivación hacia las matemáticas, la satisfacción por el aprendizaje, y el interés por la permanencia en la escuela aumentaron tras la introducción del programa.

A su vez, también pueden encontrarse ejemplos de los beneficios de las actividades de juego en materias como la educación física. Nuevamente, mediante el empleo de videojuegos relacionados con las actividades físicas y el deporte, se produjo un aumento significativo de la motivación del alumnado hacia dicha asignatura. Pese a que la motivación decayó posteriormente, sus resultados establecen un precedente dentro de la investigación sobre el juego y el aprendizaje (Sun, 2013).

No obstante, el juego no sólo influye en la motivación. Mediante programas de juego desarrollados en ámbitos extraescolares (concretamente campamentos infantiles y adolescentes), se constató un aumento tanto de la motivación como de la participación social entre los jóvenes. En consecuencia, el clima o convivencia también puede mejorar tras la implementación del juego (Zarrett, Sorensen y Skiles, 2013).

Además, el juego no tiene que ser necesariamente de carácter individual, el estudio de Gray, Sproule y Morgan (2009) reveló que las ocupaciones lúdicas por equipos son un elemento clave para la promoción de un clima tanto motivacional como participativo dentro del aula. E incluso proyectos como desarrollado por los terapeutas ocupacionales Bundy et al (2008) demuestran que la creatividad en los niños también es un factor a potenciar mediante el juego.

En conclusión, la literatura actual constata que las actividades de juego son un agente fundamental para el desarrollo integral y significativo en las etapas de la infancia y la adolescencia. Por consiguiente, se espera que dicha ocupación sea, a su vez, un agente que favorezca la satisfacción de los jóvenes dentro de los sistemas de enseñanza.

\section{Objetivos e hipótesis del proyecto}

Tomando en consideración los beneficios potenciales de actividades significativas (como el juego) en el desarrollo y aprendizaje en la infancia, la presente investigación tiene como fin:

\section{Objetivos}

Objetivo 1. Diseñar un programa de ocupaciones lúdicas que promueva la motivación del alumnado por asistir a la escuela.

Objetivo 2. Favorecer la participación social y la convivencia escolar a través del juego.

Objetivo 3. Promover, en última instancia, una mejor relación alumno-escuela y una mayor satisfacción escolar.

\section{Hipótesis}

Hipótesis 1. Los estudiantes del grupo experimental obtendrán unos niveles de motivación más elevados que el grupo de control en las medidas post-test.

Hipótesis 2. Los estudiantes del grupo experimental mostrarán una percepción más positiva del clima escolar respecto al grupo de control en las medidas post-test. 
Hipótesis 3. Los estudiantes del grupo experimental presentarán una satisfacción escolar superior al grupo de control en las medidas post-test.

\section{Método}

\section{Diseño}

El análisis y valoración de las hipótesis previamente expuestas se realizará mediante el desarrollo de un diseño de medidas repetidas. Un diseño de dos factores: uno inter-sujeto con dos niveles (intervención y no intervención) y otro intra-sujeto con cuatro niveles (cuatro medidas temporales).

Dicha investigación tendrá una duración de un curso académico completo dentro del cual se implementará el programa de juego diseñado específicamente para el estudio. Por su parte, las variables dependientes que se estudiarán son: motivación, convivencia escolar, satisfacción escolar, satisfacción general y rendimiento escolar.

\section{Participantes}

La población necesaria para el desarrollo del presente proyecto estará conformada por alumnado y profesorado de cuatro centros de enseñanza primara públicos de la ciudad de A Coruña. Tanto alumnos como profesores pertenecerán al último curso de educación primaria (sexto).

Para comprobar si se producen diferencias estadísticamente significativas antes, durante y después de la implementación del programa, se asignarán aleatoriamente dos escuelas (dos cursos de sexto de primaria) al grupo de control y otras dos al grupo experimental. Además, se realizarán las medidas pre-test en los cuatro cursos previamente a la asignación de éstos en los grupos.

\section{Instrumentos de recogida de información}

Los instrumentos seleccionados para la recogida y posterior análisis de las variables son:

El cuestionario MAPE-1. Diseñado por Alonso Tapia y Sánchez Ferrer, dicho cuestionario tiene por objetivo estudiar el grado de motivación hacia el aprendizaje. Consta de tres dimensiones pero, para el desarrollo de la investigación, se seleccionarán únicamente la dimensión 1 (orientación al resultado y evitación versus orientación al aprendizaje) y la 2 (vagancia versus disposición al esfuerzo).

La batería de instrumentos para la evaluación del clima escolar en escuelas primarias. Dicha batería consta de dos subescalas, una para valorar la percepción sobre el clima escolar y otra destinada a analizar el grado de satisfacción escolar de la persona. Este instrumento será también implementado en el profesorado para conocer su visión respecto al programa.

El índice de bienestar personal IBP. Adaptación al castellano del PWI de Cummins, Eckersley, Pallant, Van Vugt y Misajon destinada a valorar el grado de satisfacción o bienestar general en niños y adolescentes. A diferencia de la batería anterior, el IBP permite valorar los efectos del estudio no sólo en el ámbito educativo, sino sus posibles efectos indirectos en la satisfacción global de los y las participantes.

A su vez, junto con los presentes instrumentos, también se tomará la variable "rendimiento escolar” para conocer si el programa incide en otros factores que no están meramente relacionados con la motivación o convivencia.

En última instancia, las variables a considerar durante la realización de la investigación serán: motivación escolar, convivencia o clima escolar, satisfacción escolar, satisfacción general, y rendimiento académico.

\section{Técnicas de análisis de datos}

Dado que la asignación a los grupos no es aleatoria, se procederá a realizar un Análisis de Covarianza (ANCOVA) con la medida pre-test como covariable.

En caso de existir diferencias significativas se realizará un ANCOVA de medidas repetidas. En caso de no existir dichas diferencias, se implementará un Análisis de Varianza (ANOVA) de medidas repetidas para valorar las diferencias en la tendencia a través del tiempo (cuatro medidas).

A su vez, se realizará un análisis intra-grupo para valorar a cada grupo por separado mediante otro ANOVA de medidas repetidas para conocer en qué momento se produce un cambio (si se produce). Dicho análisis se realizará de forma independiente con cada variable.

Dado que es uno de los procedimientos más empleados en la investigación en ciencias sociales, el coeficiente eta-cuadrado parcial $\left(\mathrm{n}_{\mathrm{p}}{ }^{2}\right)$ será la medida utilizada para estudiar el tamaño del efecto (Sun, Pan y Wang, 2010). Por su parte, la interpretación del tamaño se utilizará el criterio de Cohen (1988) según el cual el efecto es pequeño cuando $\mathrm{n}_{\mathrm{p}}^{2}=.01(d=.20)$, mediano cuando $\mathrm{n}_{\mathrm{p}}{ }^{2}$ $=.059(d=.50)$, y grande cuando $\mathrm{n}_{\mathrm{p}}{ }^{2}=.138(d=.80)$.

\section{Plan de trabajo}

Tal y como se expuso anteriormente, la presente investigación tendrá una duración de un curso académico y su desarrollo estará dividido en diferentes etapas:

Etapa 0. Etapa pre-test que tiene lugar al comienzo del curso (la primera medida de las variables del estudio).

Etapa 1. Se corresponde con el primer trimestre. En dicha etapa se comienza a implementar el programa de juego.

Etapa 2. Con el comienzo del segundo trimestre, se realizará la segunda medida $\mathrm{y}$, posteriormente, se continuará con el programa.

Etapa 3. Al igual que en la etapa anterior, el tercer trimestre comenzará con la obtención de la siguiente medida de variables y se continuará con el programa.

Etapa 4. Al final del tercer y último trimestre se tomará la última medida de las variables coincidiendo con el final del programa.

Durante dichas etapas, el programa de juego será implementado en dos sesiones semanales de treinta minutos de duración. Cada una de estas sesiones tendrá una dinámica diferente: una destinada a desarrollar actividades de juego dirigidas por el investigador y otra 
orientada a la promoción de un juego libre y creativo por parte de los alumnos y alumnas participantes.

\section{Programa de intervención}

Para el desarrollo del estudio se ha diseñado un programa de juego específico, el Programa de Actividades de Xogo: Ven á Escola! (Programa PRAXOVES). Se trata de una herramienta piloto diseñada con el objetivo de emplear actividades u ocupaciones significativas en la infancia y la adolescencia (el juego) para promover un desarrollo satisfactorio. Además, como objetivos específicos están la promoción de la motivación por asistir a la escuela, la calidad del clima escolar, y la satisfacción final por el aprendizaje.

Los enfoques teóricos que fundamentan dicho programa son la psicología del desarrollo y la terapia ocupacional en la infancia. Las bases de la psicología son la Teoría del Aprendizaje Social de Bandura y la Teoría Atribucional de Weiner. Por su parte, las bases de terapia ocupacional son el Modelo de Exploración, Competencia y Logro de Mary Reilly y los trabajos de juego libre y creativo de Ana Bundy.

Respecto al plan de actividades, la experiencia estará dividida en dos talleres que tendrán lugar diferentes días de la semana:

Taller 1 Diseña tu aventura: Jugando con todos. Diseñado para promover la satisfacción del alumnado a través de la creación de un ambiente de convivencia y participación social positivo.

Este taller combina dos dinámicas de juego, unas dirigidas basadas en la integración y participación social $\mathrm{y}$, posteriormente, actividades de juego libre donde se facilitan materiales alternativos a los niños para que diseñen sus propias aventuras e historias de juego.

Las primeras estarán dirigidas por el coordinador del programa (siendo algunas de las actividades: Enredados, Pásame la pelota, Ordenándonos en las alturas, El ojo del grupo, etcétera.) mientras que las segundas serán dirigidas sólo por los alumnos y alumnas (algunos de los materiales facilitados son: cajas de cartón, sacos de espuma, cuerdas, pelotas, etcétera.).

Taller 2 Motívate: Aprender jugando. Dirigido a combinar los conocimientos adquiridos durante las clases con actividades de juego grupales y aumentar, con ello, la motivación por el aprendizaje.

Este taller no tiene por objetivo promover el aprendizaje del currículo académico, pero si integrar lo aprendido dentro de actividades lúdicas. Estará conformado por tres aventuras diferentes (una por trimestre) que tendrán continuidad a lo largo de las sesiones semanales donde la temáticas del aula serán el contexto de la aventura, así como parte de las actividades de ésta.

Las aventuras para cada trimestre son: La máquina del tiempo (cuyo contexto es la Historia), Animales $y$ personas (basado en las Ciencias Naturales), y Un paseo por la Tierra (centrado en la Geografía y las Ciencias Sociales).

\section{Limitaciones del estudio}

Las principales limitaciones que presenta el estudio de cara a su desarrollo son, por una parte, la adecuación de las sesiones del programa a los tiempos y horarios de los centros seleccionados.

$\mathrm{Y}$ por otro lado, el volumen de trabajo, personal y tiempo requeridos para la implementación del programa imposibilita actualmente su puesta en práctica.

\section{Plan de financiación}

Para el desarrollo de dicha investigación, los gastos estimados giran en torno a los $6680 €$. Dentro de estos gastos se tiene en consideración tanto material inventariable (ordenadores, impresora, etc.) como fungible (material de oficina, fotocopias, etc.). Además de contar con los gastos de los materiales de juego para los cuatro centros educativos.

Como posibles fuentes de financiación destacan tanto fuentes de carácter público: Premios nacionales para centros educativas (Ministerio de Educación), Plan galego de investigación e innovación (Consellería de Educación, Xunta de Galicia), o Ayudas a la investigación 2016 (Universidade da Coruña); como fuentes de financiación privadas: Becas para jóvenes investigadores (Santander Universidades), Ayudas a proyectos de iniciativa social (Obra Social La Caixa), o Proyectos de educación y formación (Fundación María José Jove).

\section{Aspectos éticos}

De cara a la realización del presente proyecto, los investigadores se comprometen a mantener el anonimato de los/las participantes según las directrices de la Ley Orgánica 15/1999 de protección de datos de carácter personal.

A su vez, se solicitará un permiso oficial de la Consellería de Cultura, Educación e Ordenación Universitaria de la Xunta de Galicia, se concertará una reunión con los centros escolares y sus respectivos ANPAs, y se facilitará un documento de consentimiento informado a cumplimentar previamente al comienzo de la investigación.

\section{Referencias}

Bundy, A., Luckett, T., Naughton, G.,Tranter, P., Wyver, S., Ragen, J., Singleton, E. y Spies, G. (2008). Playful interaction: Occupational therapy for all children on the school playground. American Journal of Occupational Therapy, 62(5), 522-527.

Cabrera, P. y Galán, E. (2002). Satisfacción escolar y rendimiento académico. Revista de Psicodidáctica, 14, 87-97.

Cohen, J. (1988). Statistical power for the behavioral sciences ( $2^{\text {nd }}$ ed.). Hillsdale, NJ: Erlbaum,.

Cornejo, R. y Redondo, J. M. (2001). El clima percibido por los alumnos de enseñanza media. Última Década, $15,11-52$.

De La Torre, C. y Godoy, A. (2002). Influencia de las atribuciones causales del profesor sobre el rendimiento de los alumnos. Psicothema, 14(2), 444-449. 
García, M. A. (2002). El bienestar subjetivo. Escritos de Psicología, 6, 18-39.

Gillet, N., Vallerand, R. y Lafrenière, M. A. (2012). Intrinsic and extrinsic school motivation as a function of age: the mediating role of autonomy support. Social Psychology Education, 15, 77-95. doi: 10.1007/s11218-011-9170-2

González, R., Valle, A., Núñez, J. C. y González-Pienda, J. A. (1996). Una aproximación teórica al concepto de metas académicas y su relación con la motivación escolar. Psicothema, 8(1), 45-61.

Gray, S., Sproule, J. y Morgan, K. (2009). Teaching team invasion games and motivational climate. European Physical Education Review, 15(1), 65-89. doi: 10.1177/1356336x09105212

Huang, Y-M., Huang, S-H. y Wu, T-T. (2014). Embedding diagnostic mechanisms in a digital game for learning mathematics. Educational Technology Research and Development, 62, 187-207. doi: 10.1007/s11423-013-9315-4

Luna, F. J. (2012). Bienestar subjetivo y satisfacción escolar en la adolescencia. (Tesis de doctorado). Universitat de Girona, Girona.

Peetsma, T. y Van der Veen, I. (2013). Influencing young adolescents' motivation in the lowest level of secondary education. Educational Review, 67(1), 97-120. doi: 10.1080/00131911.2013.830593

Regueiro, B., Suárez, N., Valle, A., Núñez, J. C. y Rosário, P. (2015). La motivación e implicación en los deberes escolares a lo largo de la escolaridad obligatoria. Revista de Psicodidáctica, 20(1), 47-63. doi: $\quad$ 10.1387/ Rev Psicodidact.12641
Ryan, R. M. y Deci, E. L. (2000). Intrinsic and extrinsic motivations: Classic definitions and new directions. Contemporary Educational Psychology, 25, 54-67. doi: 10.1006/ceps.1999.1020

Singh, K. y Junnarkar, M. (2014). Correlates and predictors of positive mental health for school going children. Personality and Individual Differences, 75, 82-87. doi: 10.1016/j.paid.2014.11.047

Sun, H. (2013). Impact of exergames on physical activity and motivation in elementary school students: A follow-up study. Journal of Sport and Health Science, 2, 138-145. doi: 10.1016/j.jshs.2013.02.003

Sun, Sh., Pan, W. y Wang, L. L. (2010). A comprehensive review of effect size reporting and interpreting practices in academic journals in education psychology. Journal of Educational Psychology, 102, 989-1004.

Valenzuela, J. (2007). Más allá de la tarea: pistas para una redefinición del concepto de motivación escolar. Educação e Pesquisa, 33(3), 409-422. doi: 10.1590/s1517-97022007000300002

Zarrett, N., Sorensen, C. y Skiles, B. (2013). Environmental and social-motivational contextual factors related to youth physical activity: Systematic observations of summer day camps. International Journal of Behavioral Nutrition and Physical Activity, 10. doi: 10.1186/1479-5868-10-63 